\title{
Typical radiological progression and clinical features of patients with coronavirus disease 2019
}

\author{
Min Wang ${ }^{1, *}$, Linghong Guo ${ }^{1, *}$, Qi Chen ${ }^{1}$, Guojin Xia ${ }^{1}$, Bo Wang ${ }^{1}$ \\ ${ }^{1}$ Department of Radiology, The First Affiliated Hospital of Nanchang University, Nanchang 330006, Jiangxi \\ Province, China \\ *Equal contribution \\ Correspondence to: Min Wang; email: wangmin df@163.com \\ Keywords: COVID-19, 2019 novel coronavirus pneumonia, radiological features, chest CT, ground-glass opacity \\ Received: March 31, $2020 \quad$ Accepted: April 11, $2020 \quad$ Published: May 2, 2020
}

Copyright: Wang et al. This is an open-access article distributed under the terms of the Creative Commons Attribution License (CC BY 3.0), which permits unrestricted use, distribution, and reproduction in any medium, provided the original author and source are credited.

\section{ABSTRACT}

We aimed to describe typical radiological features and progression of Coronavirus disease 2019 (COVID-19) patients. We reviewed the chest CT scans, laboratory findings, and clinical records of 66 COVID-19 patients who were admitted to affiliated hospitals of Nanchang university, Nanchang, China, from Jan 21 to Feb 2, 2020. CT was used to evaluate the radiological characteristics of COVID-19 patients. Only 4 patients (4/66,6\%) claimed their exposure to COVID-19 pneumonia patients. The major symptoms were fever $(60 / 66,91 \%)$ and cough $(37 / 66,56 \%)$. The predominant features of lesion were scattered $(43 / 66,65 \%)$, bilateral $(50 / 66,76 \%)$, groundglass opacity $(64 / 66,97 \%)$, and air bronchogram sign $(47 / 66,71 \%)$. Forty-eight patients $(48 / 66,73 \%)$ had more than two lobes involved. Right lower lobe $(58 / 66,88 \%)$ and left lower lobe $(49 / 66,74 \%)$ were most likely invaded. Twelve patients $(12 / 66,18 \%)$ had at least one comorbid condition. Pleural traction $(29 / 66,44 \%)$, crazy paving $(15 / 66,23 \%)$, interlobular septal thickening $(11 / 66,17 \%)$, and consolidation $(7 / 66,11 \%)$ were also observed. The typical radiology features of COVID-19 patients are scattered ground-glass opacity in the bilateral lobes. Fever and cough are the major symptoms. Evaluating chest CT, clinical symptoms, and laboratory results could facilitate the early diagnosis of COVID-19, and judge disease progression.

\section{INTRODUCTION}

Since December 2019, a series of unknown pneumonia caused by a novel coronavirus broke out in Wuhan, Hubei, China. This new coronavirus was named as severe acute respiratory syndrome coronavirus 2 (SARS-Cov-2) or 2019 novel coronavirus (2019-nCoV) [1]. The disease caused by $2019-\mathrm{nCoV}$ is coronavirus disease 2019 (COVID-19), which had been confirmed to be a global pandemic by the World health organization (WHO). By April 8 2020, more than 1, 350, 000 infected cases and 79,000 deaths have been caused by COVID-19 [2]. COVID-19 has been effectively prevented and controlled in China, Singapore, South Korea, and Japan right now, but 2019$\mathrm{nCoV}$ is spreading fast in Europe and the United State.
Obviously, the threat to the global health and economy by $2019-\mathrm{nCoV}$ will last for a long time [3, 4].

2019-nCoV, a betacoronavirus, is a member of family Coronaviridae [5]. In total, six types of coronavirus have been identified including middle east respiratory syndrome coronavirus (MERS-CoV), severe acute respiratory syndrome coronavirus (SARS-CoV), NL-63, OC-43, and 229E, among which MERS-CoV and SARS-CoV could cause severe respiratory diseases [6]. 2019-nCoV, a novel coronavirus, could interact with the human angiotensin converting enzyme 2 receptor through its spike protein [7, 8]. 2019-nCoV spread among population mainly through respiratory droplets and direct contact, and could cause several different symptoms including fever, cough, and fatigue [9]. 
Table 1. Clinical characteristics and laboratory results of patient with COVID-19 pneumonia ( $n=66$ ).

\begin{tabular}{|c|c|}
\hline Characteristic & Number (\%) \\
\hline Male & $43(65 \%)$ \\
\hline Female & $23(35 \%)$ \\
\hline$>50$ years old & $21(32 \%)$ \\
\hline$\leq 50$ years old & $45(68 \%)$ \\
\hline Exposure to COVID-19 pneumonia patients & $4(6 \%)$ \\
\hline Lived in or visited Wuhan during the epidemic & $21(32 \%)$ \\
\hline Unknown exposure & $41(62 \%)$ \\
\hline \multicolumn{2}{|l|}{ Symptoms } \\
\hline Fever & $60(91 \%)$ \\
\hline Cough & $37(56 \%)$ \\
\hline Sore throat & $17(26 \%)$ \\
\hline Sputum & $16(24 \%)$ \\
\hline Fatigue & $15(23 \%)$ \\
\hline Dyspnoea & $14(21 \%)$ \\
\hline Dizziness & $9(14 \%)$ \\
\hline Myalgia & $7(11 \%)$ \\
\hline Headache & $3(5 \%)$ \\
\hline Diarrhoea & $3(5 \%)$ \\
\hline Nausea & $3(5 \%)$ \\
\hline Rhinorrhea & $2(3 \%)$ \\
\hline \multicolumn{2}{|l|}{ C-reactive protein (mg/L; normal range $0-10)$} \\
\hline Increased & $38(58 \%)$ \\
\hline Decreased & 0 \\
\hline Normal & $28(42 \%)$ \\
\hline \multicolumn{2}{|l|}{ Leucocytes $\left(\times 10^{9}\right.$, normal range $\left.3.5-9.5\right)$} \\
\hline Increased & $1(2 \%)$ \\
\hline Decreased & $14(21 \%)$ \\
\hline Normal & $51(77 \%)$ \\
\hline \multicolumn{2}{|l|}{ Lymphocyte $\left(\times 10^{9}\right.$, normal range $\left.1.1-3.2\right)$} \\
\hline Increased & 0 \\
\hline Decreased & $29(44 \%)$ \\
\hline Normal & $37(56 \%)$ \\
\hline \multicolumn{2}{|l|}{ Comorbid conditions } \\
\hline Any & $12(18 \%)$ \\
\hline Hepatitis or liver cirrhosis & $8(12 \%)$ \\
\hline Hypertension & $4(6 \%)$ \\
\hline Diabetes & $2(3 \%)$ \\
\hline Chronic pulmonary disease & $1(2 \%)$ \\
\hline Cardiovascular disease & $1(2 \%)$ \\
\hline
\end{tabular}

Early diagnose of 2019-nCoV is important for the next isolation and treatment. However, shortage of nucleic acid detection reagent has been reported in some countries. CT characterized by convenience and accuracy plays a key role in the diagnose of respiratory diseases. CT provides a simple, direct, and convenient auxiliary diagnosis method for the patients, who cannot be tested by RT-PCR. However, there are very few studies focusing on the lung CT features of COVID-19 patients so far.

In this study, we summarized the radiological characteristics and clinical features of 66 COVID-19 patients. We aimed to unfold the typical radiology characteristics and progression of COVID-19 patients. This study may provide helpful images for early diagnose and treatment.

\section{RESULTS}

Total 66 COVID-19 pneumonia patients were admitted to three affiliated hospitals of Nanchang university between Jan 21 to Feb 2, 2020 (Table 1). Epidemiological investigation indicated that 4 (4/66, 6\%) patients had direct exposure to COVID-19 
pneumonia patients, $21(21 / 66,32 \%)$ patients lived in or visited Wuhan during epidemic, and 41 (41/66, 62\%) patients did not have obvious exposure. In this cohort, the average age of all patients was 44 years (SD 14; range 18-75), and there were 43 male patients (43/66, $65 \%)$ and 23 female patients $(23 / 66,35 \%) .45$ (45/66, $68 \%$ ) patients were younger than 50 years, and 21 $(21 / 66,32 \%)$ patients were elder than 50 years.

The most common symptoms were fever $(60 / 66,91 \%)$ and cough $(37 / 66,56 \%)$. Some other symptoms, such as sore throat $(17 / 66,26 \%)$, sputum $(17 / 66,24 \%)$, fatigue $(15 / 66,23 \%)$, and dyspnoea $(14 / 66,21 \%)$, were also observed frequently. Other non-specific symptoms included myalgia $(7 / 66,11 \%)$, headache $(3 / 66,5 \%)$, diarrhea $(3 / 66,5 \%)$, and nausea $(3 / 66,5 \%) .38(38 / 66$, $58 \%$ ) patients had a higher level of C-reactive protein, and the rest of patients were normal. The leucocytes level of $51(51 / 66,77 \%)$ patients was normal. 29 $(29 / 66,44 \%)$ patients had decreased lymphocyte level, and $37(37 / 66,56 \%)$ patients' lymphocyte count was normal. Comorbid conditions were not common in these patients, and only $12(12 / 66,18 \%)$ patients had at least one complication.
Scattered lesions found in $43(43 / 66,65 \%)$ patients were most common, and $23(23 / 66,35 \%)$ patients shown subpleural distribution. Lesion involved bilateral lungs was observed in $50(50 / 66,76 \%)$ patients. Lesion invaded more than two lobes was found in 48 (48/66, $71 \%)$ patients. Right lower lobe (58/66, 88\%) and left lower lobe $(49 / 66,74 \%)$ were most likely to be involved.

In this study, we presented some common and typical radiology changes (Figures 1 and 2). The most common radiology characteristic seen on the CT was ground-glass opacity $(64 / 66,97 \%)$. Most ground-glass opacities were characterized by scattered and bilateral lesions (Figure 1A and 1B). The CT scans of 15 $(15 / 66,23 \%)$ patients shown crazy paving (Figure 1C), and consolidation was observed in $7(7 / 66,11 \%)$ patients (Figure 2A). In addition, air bronchogram sign (47/66, 71\%, Figure 1D), pleural traction (29/66, $44 \%)$, interlobular septal thickening $(11 / 66,17 \%)$, and halo sign $(3 / 66,5 \%$, Figure $2 \mathrm{~B})$ were also observed (Table 2). Bronchiectasia was observed in the right lower lobe of one patient with bilateral ground-glass opacity (Figure 2C).
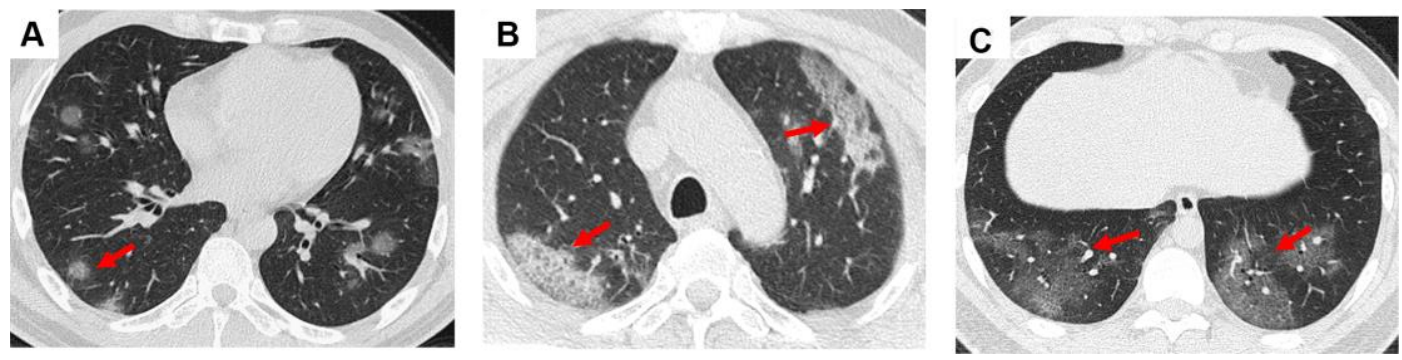

Figure 1. Ground-glass opacity and crazy paving in the CT scans of COVID-19 pneumonia patients. (A) Multiple nodular groundglass opacity scattered in both lungs of a 44-year-old male patient; (B) Mixed ground-glass opacity along the long axis of subpleural in both lungs of a 67-year-old male patient; (C) Crazy paving was observed in the bilateral lower lungs of a 67-year-old male patient at the fourth day since admission. Typical lesions were marked with red arrows.
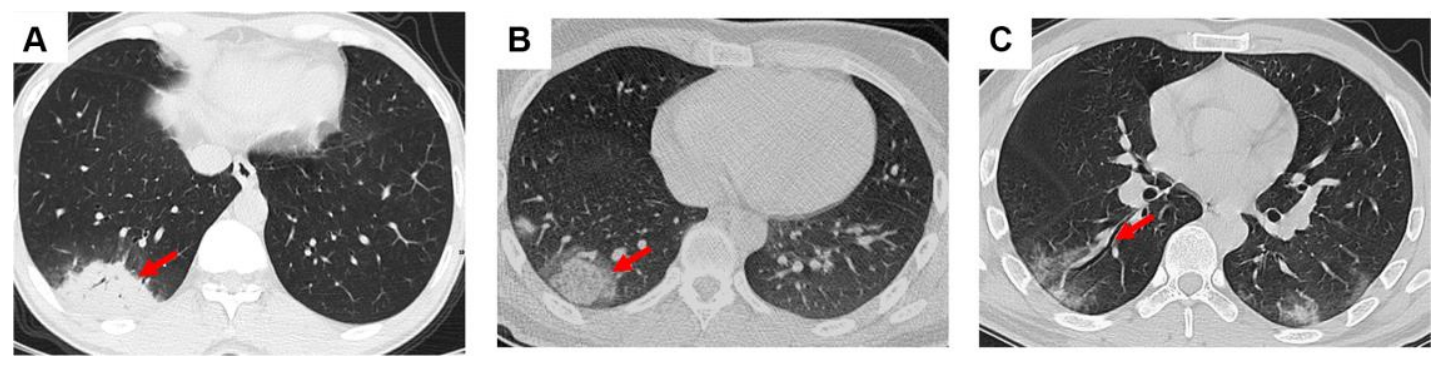

Figure 2. Consolidation, halo sign, and bronchiectasia in the CT scans of COVID-19 pneumonia patients. (A) Consolidation accompanying air bronchogram sign was found in the right lower lobe of a 46-year-old male patient; (B) Halo sign was observed in the right lower lobe of a 18-year-old male patient; (C) Bronchiectasia was observed in the right lower lobe of a 30-year-old male patient with bilateral ground-glass opacity. Typical lesions were marked with red arrows. 
Table 2. CT findings of patient with COVID-19 pneumonia ( $n=66)$

\begin{tabular}{lc}
\hline CT features & Number (\%) \\
\hline Distribution & $23(35 \%)$ \\
Subpleural & 0 \\
Central & $43(65 \%)$ \\
Scattered & \\
Number of lobes involved & $13(20 \%)$ \\
1 & $5(8 \%)$ \\
2 & $10(15 \%)$ \\
3 & $11(17 \%)$ \\
4 & $27(41 \%)$ \\
5 & $48(73 \%)$ \\
More than two lobes involved & \\
Lobe of lesion distribution & $42(64 \%)$ \\
Right upper lobe & $37(56 \%)$ \\
Right middle lobe & $58(88 \%)$ \\
Right lower lobe & $44(67 \%)$ \\
Left upper lobe & $49(74 \%)$ \\
Left lower lobe & $50(76 \%)$ \\
Lesion involved bilateral lungs & $16(24 \%)$ \\
Lesion involved unilateral lung & \\
Lesion characteristics & $64(97 \%)$ \\
Ground-glass opacity & $15(23 \%)$ \\
Crazy paving & $7(11 \%)$ \\
Consolidation & \\
Lesion shape & $66(100 \%)$ \\
Patch & $13(20 \%)$ \\
Circular & $12(18 \%)$ \\
Reticular spline & \\
Other signs in the lesion & $47(71 \%)$ \\
Air bronchogram sign & $29(44 \%)$ \\
Pleural traction & $11(17 \%)$ \\
Interlobular septal thickening & $3(5 \%)$ \\
Vacuole Halo sign & \\
Other findings & $4(6 \%)$ \\
Pulmonary emphysema & $2(3 \%)$ \\
Pulmonary fibrosis & $1(2 \%)$ \\
Pleural effusion & $1(2 \%)$ \\
Tuberculosis & $1(2 \%)$ \\
\hline & \\
\hline & \\
\hline
\end{tabular}

By Mar 23, 2020, $60(60 / 66,91 \%)$ patients had been discharged. $6(6 / 66,9 \%)$ patients were still in hospital, and two patients had died because of ARDS. Patient 1, 78-year-old man with hypertension, who died on day 15 after admission (Figure 1B). Patient 2, 47-year-old man with type 2 diabetes, whose $\mathrm{CT}$ scan presented rapid radiology progression (Figure 3A, 3B). The radiological change of COVID-19 pneumonia develops fast during the first seven days (Figure 3C, 3D). Some of patch lesion could be absorbed and change into reticular spline lesion (Figure 4A, 4B). Meanwhile, some patients achieved rapid recovery with significant improvement of CT sign (Figure 4C, 4D) and clinical symptoms. We also did some CT follow-up scans for few patients, which showed the aggravated progression of disease since admission and rapid recovery after treatment (Figures 5 and 6). Disappearance of lesions and significant improvement of clinical symptoms were observed in two patients (Figure 5: a 54-year-old male patient; Figure 6: a 54-year-old female patient).

\section{DISCUSSION}

2019-nCoV, an enveloped positive-sense RNA virus, is the seventh member of the coronaviridae family [10]. It is estimated that 2019-nCoV could cause 1\%-6\% mortality rate depending on different regions, which is lower than MERS-CoV (10\%) and SARS-CoV (37\%) 

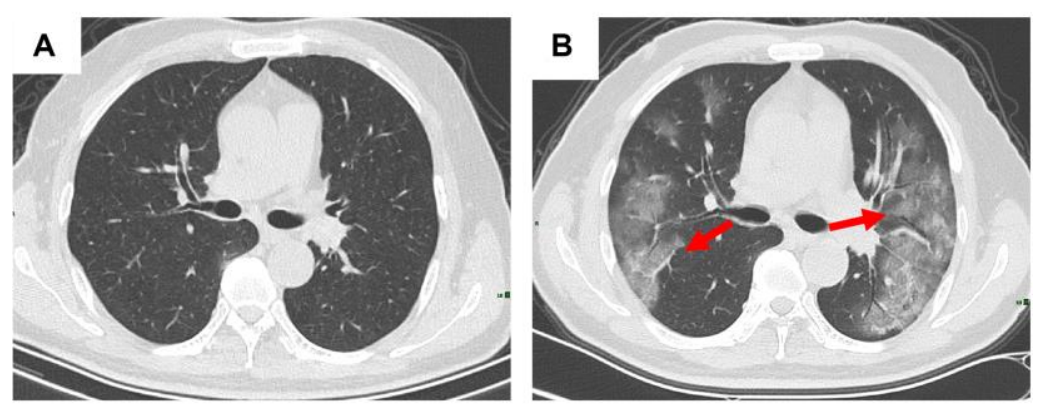

2020/01/28

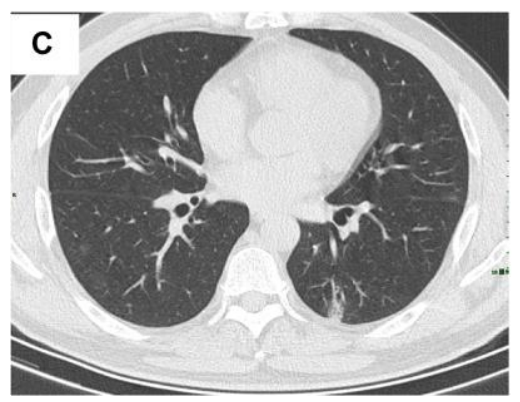

2020/02/02

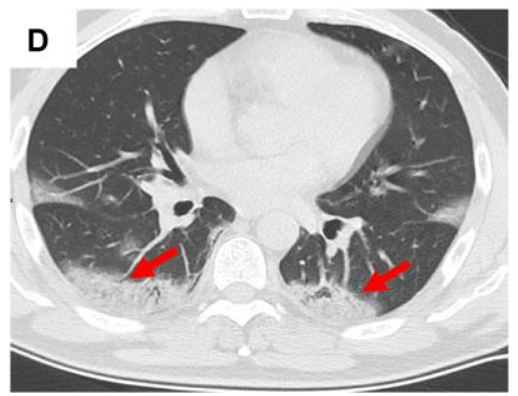

Figure 3. Radiological worsen progression of two COVID-19 pneumonia patients. (A, B): Bilateral, large, and multiple ground-glass opacity was observed in a 47-year-old male patient with type 2 diabetes after 8 days since admission; (C, D) Consolidation accompanying air bronchogram were found in the bilateral lower lungs of a 29-year-old male patient after 5 days since admission. Typical lesions were marked with red arrows.

2020/01/31

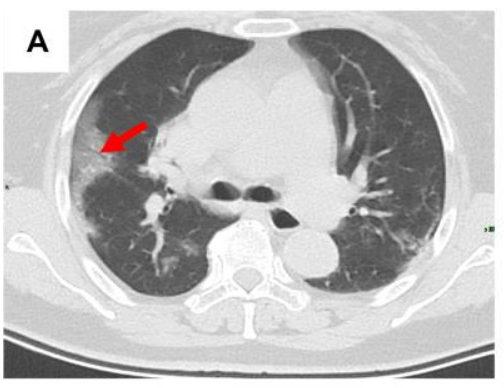

2020/01/28

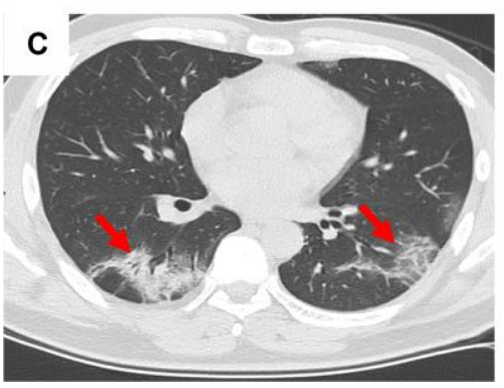

2020/02/06

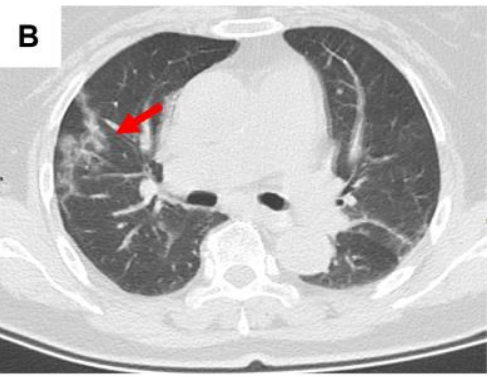

2020/02/02

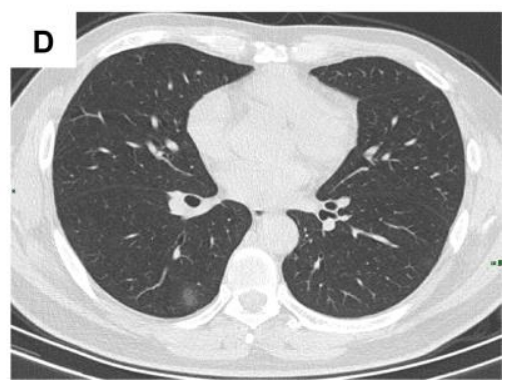

Figure 4. Radiological improvement of two COVID-19 pneumonia patients. (A, B) Patch lesions were absorbed and changed into reticular spline ones (a 31-year-old female patient); (C-D) Significant improvement of CT sign was achieved in a 22-year-old male patient. Typical lesions were marked with red arrows. 
[5, 11], but the high infectivity of the pathogen has caused a global pandemic. 2019-nCoV has became a huge threat for the global health, economic development, and social stability.

Previous study indicated that old age population with comorbidities were susceptible to infection of 2019nCoV [12]. In our cohort, there were $45(45 / 66,68 \%)$ patients under 50 years-old, and only $12(12 / 66,8 \%)$ patients had at least one comorbid condition. Small cohorts and differences in demographic characteristics might account for this discrepancy. Previous study suggested that $73 \%$ (30/41) patients were male [1], which is inconsistent with another study [13]. In our study, male infected patients account for $65 \%$ (43/66). The difference of gender distribution might also due to small cohorts. By Mar 23, 2020, two patients (78 and 47 years old, respectively) in this study had died, and both had comorbid conditions.

It is worth mentioning that 41 patients $(41 / 66,62 \%)$ patients had no obvious exposure history indicating that they might be infected by latent infection patients.
Latent infection should attract the attention of people, because the clinical appearance of latent infection patients is not consistent with real disease progression. Meanwhile, the latent infection patients indeed have infectivity. When participating in group activities or gathering, wearing mask should be an effective method to prevent infectivity by asymptomatic patients. Some countries such as India and Indonesia have a large population and the medical condition of them is not optimistic. For the people who lack sufficient medical protection, it is effective to prevent and control virus spreading by avoiding gathering, wearing mask in the crowd, regular ventilation at home.

Fever and cough were the most common symptoms in the COVID-19 patients. Self-isolation and wearing mask are still effective and economic method for fever people who have mild symptoms, but if symptoms aggravate, professional and medical measures should be taken because of the high mortality rate.

Due to special structure, right lower lobe and left lower lobe were most commonly involved, which is in line
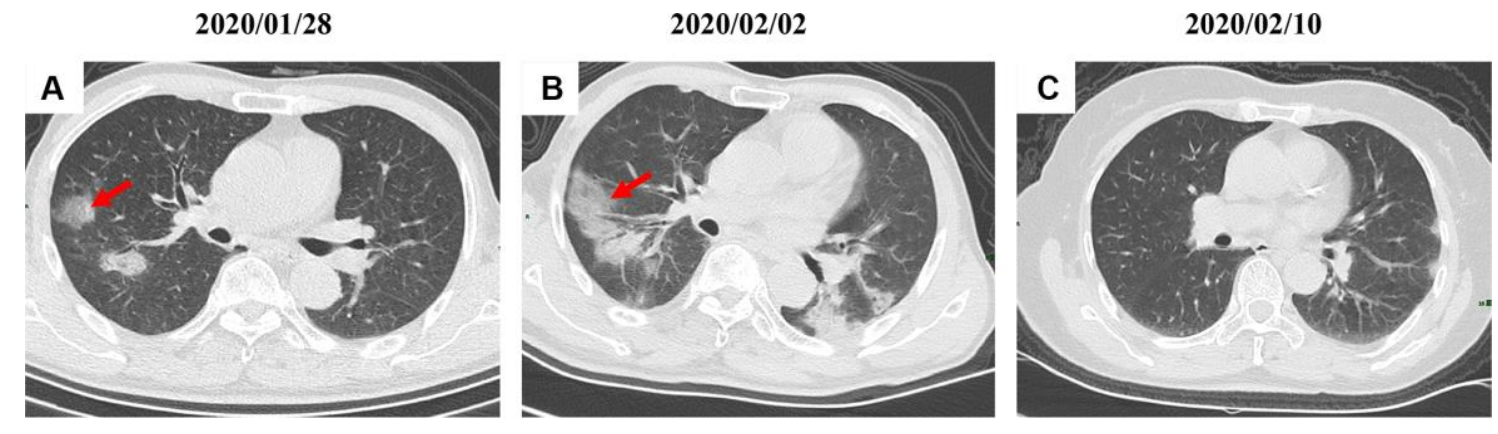

Figure 5. A serial CT images after admission of a 54-year-old male patient. (A) Patch ground-glass opacity was observed in the middle right lobe. (B) 5 days later, significant larger patch ground-glass opacities were observed in bilateral lungs. (C) Follow-up CT scans on day 13 after admission show a remarkable improvement. Typical lesions were marked with red arrows.

2020/01/23

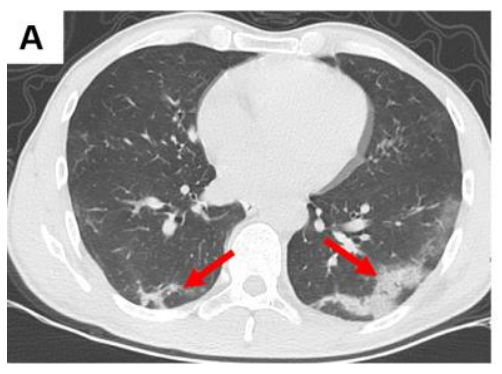

2020/01/31

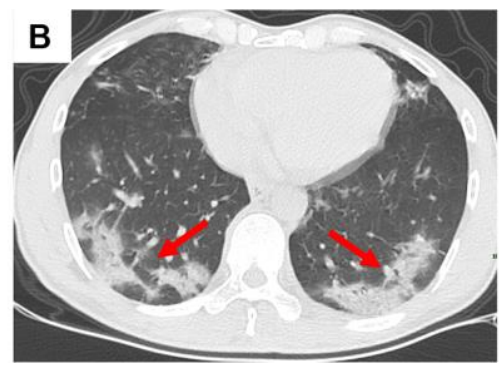

2020/02/12

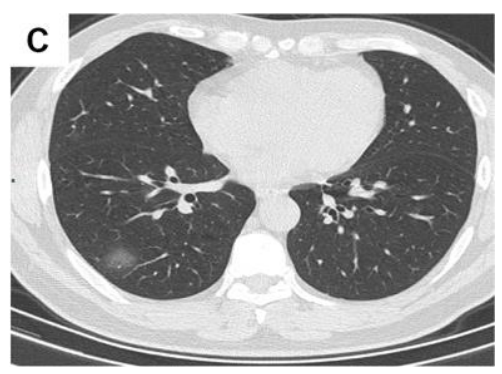

Figure 6. A serial CT images after admission of a 54-year-old female patient. (A) Patch ground-glass opacity mainly located in the left lower lobe. (B) Significant larger patch ground-glass opacities were observed in both lower lobes after 8 days. (C) Follow-up CT scans on day 20 after admission show a remarkable improvement. Typical lesions were marked with red arrows. 
with previous study [13]. Most COVID-19 patients presented bilateral lungs lesion with scattered distribution. However, unilateral lesion is more common in the early infection stage of MERS-CoV and SARS-CoV $[14,15]$. The most common image feature was ground-glass opacity, which was found in 64 (64/66, 97\%) patients. Other features such as crazy paving, consolidation, air bronchogram sign, and pleural traction were also observed. However, these radiological characteristics could be found in other viral pneumonia caused by MERS-CoV, SARS-CoV, and adenovirus.

RT-PCR has been viewed as the gold standard for COVID-19 pneumonia diagnosis. While, many countries are facing the shortage of nucleic acid test reagent. Meanwhile, the it nucleic acid test costs at least 4-5 hours including throat swab collection, RNA extraction, and RT-PCR. Chest CT could provide effective and fast evidence for the clinical diagnosis of COVID-19 pneumonia. Imaging findings could also indicate the prognosis. The radiological features of some patients might worsen fast indicating a poor prognosis (Figure 3A, 3B).

Our study had some limitations. Due to short time for data collection, we did not conduct long-term follow-up $\mathrm{CT}$, which is necessary to evaluate the prognosis of patients. In addition, we did not systematically investigate the radiology progression of patients, which could help to judge disease course of COVID-19 pneumonia.

In summary, the typical radiology features of COVID19 pneumonia were characterized by bilateral and scattered ground-glass opacity accompanying with air bronchogram sign, and predominant lesion location in the left lower lobe and right lobe. Sometimes, the clinical symptoms were not consistent with imaging features indicating that asymptomatic patients may account for a certain proportion. Therefore, CT should be an effective, fast, and simple method for the screening, diagnose, and treatment of COVID-19 pneumonia.

\section{MATERIALS AND METHODS}

\section{Patients}

The retrospective study was approved by the ethical committee of affiliated hospitals of Nanchang university. The written informed consent of this research has been waived by the ethics committee of our hospital for the reason that there is no potential risk and this is a retrospective study. The COVID-19 patients identified by RT-PCR or nest-generation sequencing were admitted from Jan 21 to Feb 2, 2020.
A total of 66 patients were enrolled (43 men and 23 women, $18-75$ years old, average age: 44 years). Throat swab samples were collected by experienced nurses, and total RNA extraction was conducted using TRIzol reagent (Thermo scientific, CA, USA). According to previous study [13], related primers (forward primer: $5^{\prime}-$ TCAGAATGCCAATCTCCCCAAC-3'; reverse primer: 5'-AAAGGTCCACCCGATACATTGA-3') were used to detect SARS-CoV-2.

\section{CT data acquisition}

All patients were examined by CT for 2-6 times at different time points. The patients in the supine position were scanned using Siemens Emotion 16 (Siemens Healthineers, Forchheim, Germany), Phillips iCT 256 (Phillips Healthcare, Andover, MA, USA), or GE revolution frontier (GE Healthcare, Issaquah, WA, USA). Scans were conducted from the apex of lung to the base of lung on the condition that patients were instructed to hold breath during examination. The following scan parameters were used: tube voltage 120 $\mathrm{kV}$, tube current 70-168mAs, pitch 08-1.2 mm, slice thickness $5 \mathrm{~mm}$, matrix $512 \times 512$, FOV $55 * 35 \mathrm{~cm}$, axial reconstruction image layer thickness $1-1.5 \mathrm{~mm}$. Three experienced radiologists blinded to nucleic acid results of patients, reviewed all CT scans.

\section{Data analysis}

Data analysis was performed on SPSS 22.0 (IBM, Armonk, NY, USA). Categorical variables were presented as number (\%), and continuous variables were shown as a range.

\section{Abbreviations}

COVID-19: Coronavirus disease 2019; WHO: World health organization; SARS-Cov-2: severe acute respiratory syndrome coronavirus 2; 2019-nCoV: 2019 novel coronavirus; MERS-CoV: Middle east respiratory syndrome coronavirus; SARS-CoV: Severe acute respiratory syndrome coronavirus.

\section{CONFLICTS OF INTEREST}

These authors declare no conflicts of interest.

\section{REFERENCES}

1. Huang $C$, Wang $Y$, Li X, Ren L, Zhao J, Hu Y, Zhang L, Fan G, Xu J, Gu X, Cheng Z, Yu T, Xia J, et al. Clinical features of patients infected with 2019 novel coronavirus in Wuhan, China. Lancet. 2020; 395:497-506. https://doi.org/10.1016/S0140-6736(20)30183-5 PMID:31986264 
2. WHO. Novel Coronavirus (COVID-19). Situation; 2020.

3. Khot WY, Nadkar MY. The 2019 Novel Coronavirus Outbreak - A Global Threat. J Assoc Physicians India. 2020; 68:67-71.

PMID: $\underline{32138488}$

4. Zu ZY, Jiang MD, Xu PP, Chen W, Ni QQ, Lu GM, Zhang LJ. Coronavirus Disease 2019 (COVID-19): A Perspective from China. Radiology. 2020; 200490. [Epub ahead of print].

https://doi.org/10.1148/radiol.2020200490

PMID:32083985

5. Xu X, Yu C, Qu J, Zhang L, Jiang S, Huang D, Chen B, Zhang Z, Guan W, Ling Z, Jiang R, Hu T, Ding Y, et al. Imaging and clinical features of patients with 2019 novel coronavirus SARS-CoV-2. Eur J Nucl Med Mol Imaging. 2020; 47:1275-80.

https://doi.org/10.1007/s00259-020-04735-9

PMID: 32107577

6. $X u X W$, Wu XX, Jiang XG, Xu KJ, Ying $\amalg, M a C L, ~ L i ~ S B$, Wang HY, Zhang S, Gao HN, Sheng JF, Cai HL, Qiu YQ, Li LJ. Clinical findings in a group of patients infected with the 2019 novel coronavirus (SARS-Cov-2) outside of Wuhan, China: retrospective case series. BMJ. 2020; 368:m606.

https://doi.org/10.1136/bmj.m606

PMID:32075786

7. Hoffmann $\mathrm{M}$, Kleine-Weber $\mathrm{H}$, Schroeder $\mathrm{S}$, Krüger $\mathrm{N}$, Herrler T, Erichsen S, Schiergens TS, Herrler G, Wu NH, Nitsche A, Müller MA, Drosten C, Pöhlmann S. SARSCoV-2 Cell Entry Depends on ACE2 and TMPRSS2 and Is Blocked by a Clinically Proven Protease Inhibitor. Cell. 2020; 181:271-280.e8.

https://doi.org/10.1016/i.cell.2020.02.052

PMID:32142651

8. Yan R, Zhang Y, Li Y, Xia L, Guo Y, Zhou Q. Structural basis for the recognition of SARS-CoV-2 by full-length human ACE2. Science. 2020; 367:1444-48. https://doi.org/10.1126/science.abb2762 PMID:32132184

9. Song F, Shi N, Shan F, Zhang Z, Shen J, Lu H, Ling $Y$, Jiang Y, Shi Y. Emerging 2019 Novel Coronavirus (2019nCoV) Pneumonia. Radiology. 2020; 295:210-17. https://doi.org/10.1148/radiol.2020200274 PMID:32027573

10. Bai HX, Hsieh B, Xiong Z, Halsey K, Choi JW, Tran TM, Pan I, Shi LB, Wang DC, Mei J, Jiang XL, Zeng QH, Egglin TK, et al. Performance of radiologists in differentiating COVID-19 from viral pneumonia on chest CT. Radiology. 2020; 200823. [Epub ahead of print]. https://doi.org/10.1148/radiol.2020200823 PMID:32155105

11. Xu YH, Dong JH, An WM, Lv XY, Yin XP, Zhang JZ, Dong L, Ma X, Zhang HJ, Gao BL. Clinical and computed tomographic imaging features of novel coronavirus pneumonia caused by SARS-CoV-2. J Infect. 2020; 80:394-400.

https://doi.org/10.1016/j.jinf.2020.02.017 PMID:32109443

12. Tian S, Hu N, Lou J, Chen K, Kang X, Xiang Z, Chen H, Wang D, Liu N, Liu D, Chen G, Zhang Y, Li D, et al. Characteristics of COVID-19 infection in Beijing. J Infect. 2020; 80:401-06. https://doi.org/10.1016/j.jinf.2020.02.018 PMID: $\underline{32112886}$

13. Shi $H$, Han $X$, Jiang $N$, Cao $Y$, Alwalid O, Gu J, Fan $Y$, Zheng $C$. Radiological findings from 81 patients with COVID-19 pneumonia in Wuhan, China: a descriptive study. Lancet Infect Dis. 2020; 20:425-34. https://doi.org/10.1016/S1473-3099(20)30086-4 PMID:32105637

14. Ooi GC, Daqing M. SARS: radiological features. Respirology. 2003 (Suppl ); 8:S15-19. https://doi.org/10.1046/i.1440-1843.2003.00519.x PMID:15018128

15. Das KM, Lee EY, Langer RD, Larsson SG. Middle East Respiratory Syndrome Coronavirus: What Does a Radiologist Need to Know? AJR Am J Roentgenol. 2016; 206:1193-201. https://doi.org/10.2214/AJR.15.15363

PMID:26998804 